\title{
Human Aspects of Visualization
}

\author{
Achim Ebert $^{1}$, Alan Dix $^{2}$, Nahum Gershon ${ }^{3}$, and Margit Pohl ${ }^{4}$ \\ ${ }^{1}$ German Research Center for Artificial Intelligence (DFKI), Kaiserslautern, Germany \\ ebert@dfki.de \\ ${ }^{2}$ Lancaster University, Lancaster, UK \\ alan@hcibook.com \\ ${ }^{3}$ The MITRE Corp., McLean, VA, USA \\ gershon@mitre.org \\ ${ }^{4}$ Vienna University of Technology, Vienna, Austria \\ margitaigw.tuwien.ac.at
}

\section{Goals and Issues}

The quantity of data available in our modern information society is ever-growing. It is becoming unfeasible for any one person to oversee, much less understand the overwhelming amount of unstructured, multidimensional information he or she is confronted with every day. The human visual system, the "mind's eye", relies in large part on the eye and on the processing and the interpretation of the information processed by the brain. Visualization utilizes both.

Interactive visualization methods can increase cognitive resources by providing an additional, external visual resource to the human memory. They may reduce the amount of searching and ease the recognition of patterns as well as enhance understanding of relationships in large amounts of data and information. In addition, they provide a medium that enables the user to have a representation of information that he or she can quickly and easily modify, restructure or consider from a different perspective. This ability to manipulate the data is of extreme importance especially for analytical reasoning and sense-making. Virtually all sciences and many industries rely on this ability to identify methods and models, which can turn data into reliable and provable knowledge.

Research in HCI and visualization has produced numerous forms of visualization and methods for interacting with visualizations of large data sets. There is longstanding design advice for both static and dynamic visualization, and many user studies of particular visualization and interaction methods. However, systematic understanding of the interaction issues with visualization and the human cognitive and perceptual processes involved is perhaps less well developed. Knowledge about human perception can help to define a more systematic framework informing the design of Information Visualization tools.

Most visualizations involve some level of computation, from proportions in pie charts, to time series analysis in sales forecasting. Sometimes this is for data reduction, sometimes to reveal new or different aspects of data that are hard to see with the raw data. In complex domains, such as business strategy, environmental monitoring, network management, and security, more complex forms of processing are being used in conjunction with visualization; this area where visualization and complex data 
manipulation meet has become known as visual analytics. Even simple forms of data manipulation such as averages or clustering have issues of interpretation (e.g. visualizing uncertainty, loss of outliers), but as more complex statistical or machine learning techniques are involved how can users make sense of the derived data or control the parameters of the underlying computational processes? On the other hand, appropriate visualizations have the potential to help users make sense of these complex algorithms.

In addition, the increasing graphical processing power of PCs has fuelled a powerful demand for larger and more capable display devices whilst, the proliferation of mobile devices requires visual applications on very small screens. With these smallor large-scale displays, assumptions of the normal desktop metaphor no longer apply and mice and keyboards, designed for desktop interaction, become unsatisfactory; all calling for novel interactions devices and techniques.

\section{The Workshop}

This workshop, co-organized by IFIP WG TC13.7 on "HCI and Visualization" and the European VisMaster Coordinated Action, seeks to survey and expand our understanding of the cognitive and perceptual issues of interactive visualization and visual analytics and to bring together researchers interested in these issues. It will outline the research required to understand which aspects of analysis most closely match human capabilities, and how interactive visual support should be designed and adapted to make optimal use of human capabilities, in terms of information perception and processing. This is a challenging agenda and will need to take into account many factors including user role, tasks, collaborations, interests and previous knowledge.

Topics of interest include: models of visualization and interaction, perceptual aspects (visual discrimination, gestalt, etc.), visualization of machine learning, visualizing uncertainty, studies of use of visualization systems, distributed cognition and visualization, social and collaborative aspects of visualization and interaction, organizational and political context of visualization, display technology, interactive mobile visualization, usability and evaluation aspects.

The workshop goals require the cooperation of scientists from different disciplines addressing aspects of visualization and analysis. This will enable a mapping between foundational theories in the different research areas to support ongoing collaboration between researchers in these fields. This will include those already working at the intersection of HCI, Visualization and Visual Analytics and also related areas such as data mining, data management, perception and cognition.

Further information both before and after the workshop can be found on the workshop website at http://www.hciv.de/interact09. 